\title{
EUCALYPTUS VIM INALIS LABILL. (MYRTACEAE) NATURALIZADA EN LA REPÚBLICA ARGENTINA
}

\author{
Rosa L. Scaramuzzino', Carlos O. D'Alfonso', Juan F. Bardi', Marcelo L. Gandini ${ }^{1,2}$ \& Bruno D. Lara ${ }^{1,3}$ \\ ${ }^{1}$ Facultad de Agronomía, Universidad Nacional del Centro de la Provincia de Buenos Aires, República de Italia 780, \\ (7300) Azul, Buenos Aires, Argentina; rosas@faa.unicen.edu.ar (autor corresponsal). \\ ${ }^{2}$ Comisión Investigaciones Cientificas de la Provincia de Buenos Aires (CIC). \\ ${ }^{3}$ Consejo Nacional de Investigaciones Cientificas y Técnicas (CONICET).
}

\begin{abstract}
Scaramuzzino, R. L.; C. O. D’Alfonso; J. F. Bardi; M. L. Gandini \& B. D. Lara. 2019. Eucalyptus viminalis Labill. (Myrtaceae) naturalized in Argentina. Darwiniana, nueva serie 7(2): 342-351.

Eucalyptus is a genus of trees native in Oceania, with five species previously cited as adventive for the Argentinean Flora. In this paper, spontaneous populations of E. viminalis in Argentina are reported for the first time. Specimens were collected at Sierras de Azul, Tandilia System, province of Buenos Aires, and adjacent plain sectors. A description of the morphology of the species and habitat are presented, together with a map indicating the geographical position of the studied populations in Buenos Aires, and images showing the areal expansion of a population in a hill of the Tandilia System. Complementary, an identification key for naturalized Eucalyptus species inhabiting Argentina is provided.
\end{abstract}

Keywords. Alien species; Argentinean Flora; Eucalyptus; Myrtaceae; Tandilia System.

Resumen. Scaramuzzino, R. L.; C. O. D'Alfonso; J. F. Bardi; M. L. Gandini \& B. D. Lara. 2019. Eucalyptus viminalis Labill. (Myrtaceae): naturalizada en la República Argentina.Darwiniana, nueva serie 7(2): 342-351.

Eucalyptus es un género de árboles nativos de Oceanía, con cinco especies previamente citadas como adventicias para la flora argentina. En este trabajo se informa por primera vez sobre la presencia de poblaciones espontáneas de E. viminalis en Argentina. Se recolectaron especímenes en las Sierras de Azul, Sistema de Tandilia, provincia de Buenos Aires, y sectores de llanura adyacentes. Se aporta una descripción de su morfología, hábitat, un mapa de la posición geográfica de las poblaciones estudiadas en Buenos Aires e imágenes que ilustran la ampliación de área de una población en un cerro en el Sistema de Tandilia. Adicionalmente, se presenta una clave para diferenciar las especies espontáneas de Eucalyptus que habitan en la Argentina.

Palabras clave. Especies exóticas; Eucalyptus; Flora Argentina; Myrtaceae; Sistema de Tandilia.

\section{INTRODUCCIÓN}

El género Eucalyptus L'Hér. comprende aproximadamente 600 especies nativas de Oceanía, especialmente de Australia y Tasmania (Rotman, 2000), con excepción de algunas especies tropicales: E. deglupta Blume que es endémico de las islas Indonesias de Sulawesi y Ceram, Mindanao en el sur de Filipinas y norte de Nueva Guinea; E. wetarensis L. D. Pryor y E. urophylla S. T. Blake que se encuentran en Timor y las islas cercanas. (Granados-Sánchez et al., 2007). 
Numerosas especies se cultivan o se han ensayado para cultivo en diversos países de todos los continentes (FAO, 1981; Rejmánek \& Richardson, 2011) con fines forestales (madera para aserrados, celulosa para la industria papelera, cortinas rompeviento), ornamentales, apícolas, medicinales, para extracción de aceites esenciales y elaboración de carbón vegetal $\mathrm{y}$ otros combustibles (Chippendale, 1988; SAGYP-INTA, 1995). Alrededor de 70 especies de Eucalyptus y géneros relacionados se han naturalizado (Rejmánek \& Richardson, 2011), algunas en otras regiones de Oceanía como Nueva Zelanda (Webb et al., 1988), también en países europeos como España y Portugal (Paiva, 1997); en Sudáfrica, donde algunas especies han sido catalogadas como invasoras y otras como naturalizadas o casuales (Henderson, 2007); en América del Norte, por ejemplo en California, de las 202 especies cultivadas, se han naturalizado 18 (Ritter \& Jost, 2009); en América del Sur E. camaldulensis Dehnh. y E. globulus Labill. han sido incluidas para la flora chilena (Rodriguez et al., 2018).

En la Argentina se cultivan más de 70 especies (Dimitri, 1988). Aunque han existido referencias a la naturalización de algunas de ellas desde hace décadas (Cozzo, 1964), sólo en los últimos 20 años se han incorporado a la flora argentina cinco especies (Flora Argentina, 2019): E. grandis W. Hill ex Maiden y E. quadrangulata H. Deane \& Maiden en Jujuy (Rotman, 2000), E. cinerea F. Muell. ex Benth., E. sideroxylon A. Cunn. \& Woolls y E. tereticornis Sm. en Córdoba (Cantero et al., 2016). A su vez se ha considerado casual a E. camaldulensis Dehnh. en Santa Fe (Bedetti et al., 2017) y también ha sido mencionada para La Pampa (Steibel et al., 2000), Córdoba (Giorgis \& Tecco, 2014) y Buenos Aires (Zalba \& Villamil, 2002).

Eucalyptus viminalis Labill. es nativa del sudeste de Australia y Tasmania. Cultivada en varios países por sus usos y por su resistencia a las heladas, se ha naturalizado en Estados Unidos de Norteamérica en California (Ritter \& Yost, 2009) y en Hawaii (NMNH, 2019), en el este de África (Verdcourt, 2001) y en Nueva Zelanda (Webb et al., 1988). En Uruguay fue introducida en 1853 (Brussa, 1994).
Probablemente se cultive desde hace 150 años en la Argentina, donde también se han observado individuos creciendo espontáneamente. En la provincia de La Pampa es una de las especies de árboles más cultivada en el nordeste y centro, donde es también espontánea (Steibel et al., 2000). En la provincia de Buenos Aires se observó por primera vez su reproducción natural en la localidad de La Larga (partido de Daireaux), a partir de semillas caídas de las plantas madres (Mangieri \& Dimitri, 1961). Posteriormente en Huetel, partido de 25 de Mayo, después de incendios y lluvias se originaron densos repoblados (Cozzo, 1964; 1976). En Sierra de la Ventana se distribuyen alejados de las plantas madres (Zalba \& Villlamil, 2002). En el Sistema de Tandilia y zonas de llanura adyacentes se han efectuado observaciones, registros y mapeo de poblaciones naturalizadas de E. viminalis en los últimos 20 años (Bardi et al., 2012). En las sierras ocupa los roquedales, por lo tanto compite con la flora nativa petrofítica.

La capacidad de invasión de las especies de este género ha sido discutida por la limitada dispersión de las semillas, alta mortalidad de las plántulas y falta de ectomicorrizas compatibles (Rejmánek \& Richardson, 2011). A su vez para algunas especies se ha mencionado la necesidad de disturbios como inundaciones estacionales o incendios para su establecimiento. En cuanto a su dispersión a distancias alejadas, si bien el viento transporta las semillas, éstas carecen de estructuras especiales para ese fin (Zalba \& Villamil, 2002). Pero, aunque no se distribuyan a grandes distancias de la planta madre, pueden transformar los ambientes en los que se establecen, dado que pueden consumir gran cantidad de agua y ser causantes de la salinización de los suelos (Jobbágy \& Jackson, 2004; Jobbágy et al, 2006), por estas razones está siendo estudiado su sistema radical. Por ello es importante que se conozca que además de los ejemplares cultivados que poseen tratamiento de vivero para sus raíces, es factible ubicar en las plantaciones y sus alrededores ejemplares provenientes de semilla que no han sido sometidos a tratamientos silviculturales. Por lo tanto, es relevante el conocimiento de todas las especies de Eucalyptus naturalizadas en la Argentina. 


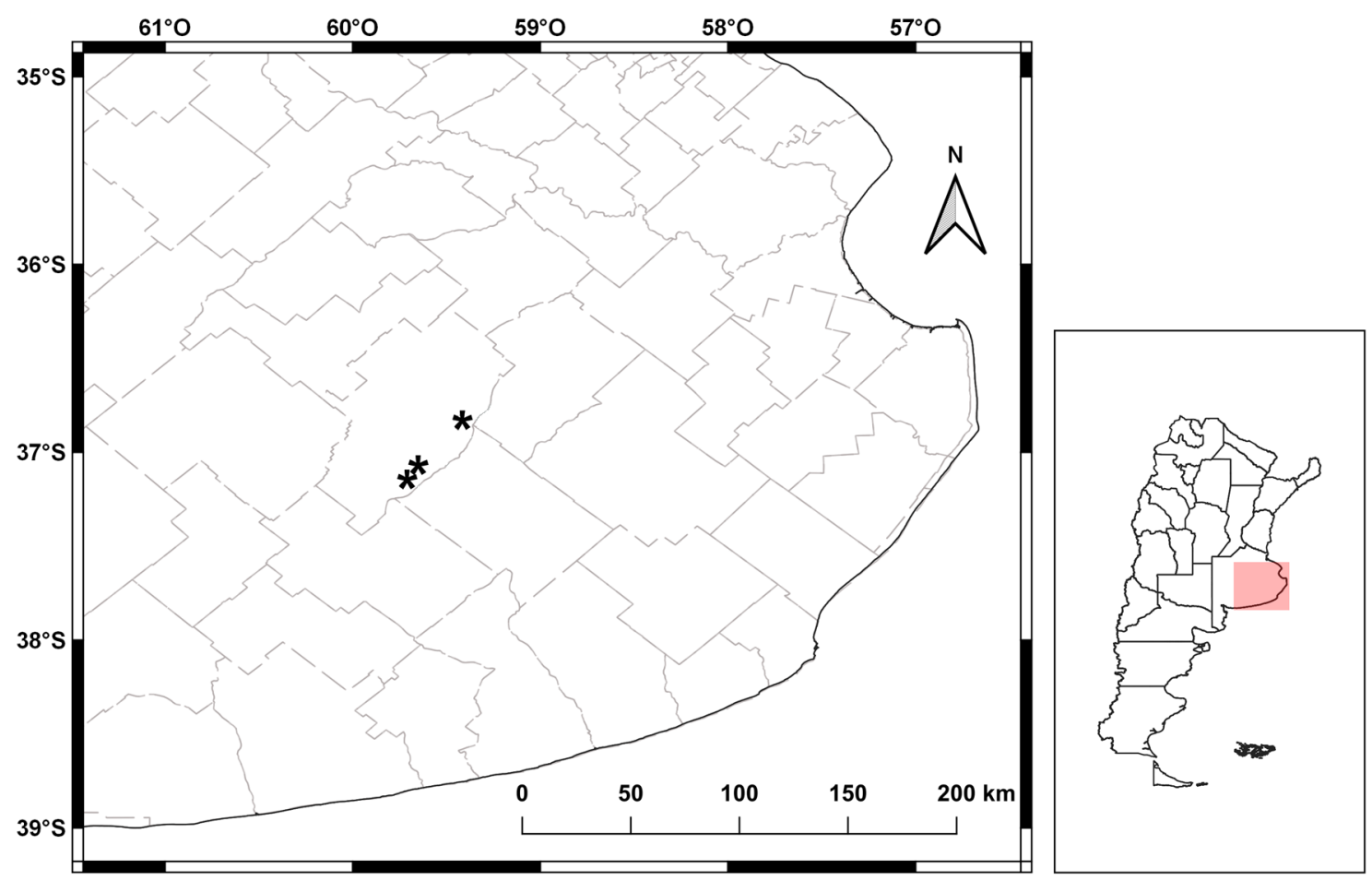

Fig. 1. Sitios de colección de Eucalyptus viminalis en la provincia de Buenos Aires, Argentina.

En el presente trabajo se cita a Eucalyptus viminalis como naturalizada en la Argentina; además, se aportan datos morfológicos, fotografías de campo, características sobre su hábitat y un mapa con la ubicación de la población hallada en el Sistema orográfico de Tandilia. Adicionalmente, se analizó mediante fotografías áreas e imágenes satelitales la ampliación del área de distribución a escala local en un cerro, donde E. viminalis modificó notoriamente el paisaje. Estos datos, junto con las citas anteriores sobre la presencia de esta especie en forma espontánea en La Pampa (Steibel et al., 2000) y Buenos Aires (Mangieri \& Dimitri, 1961; Cozzo, 1964; 1976; Zalba \& Villlamil, 2002), permiten reforzar su categorización como naturalizada (Pyšek et al., 2004) e incrementan el conocimiento de la distribución de esta especie en la Argentina. Por otro lado, se aporta una clave para diferenciar a Eucalyptus viminalis de las restantes especies de este género espontáneas en la Argentina.

\section{MATERIALES Y MÉTODOS}

El material analizado en este trabajo se recolectó en 1999 y 2019 en las Sierras de Azul, Sistema de Tandilia, provincia de Buenos Aires, en un cerro (37'3'27's, $\left.59^{\circ} 40^{\prime} 26^{\prime} \mathrm{W}\right)$, piedemonte y zonas de llanura próximas al Arroyo de los Huesos (Fig. 1). Los ejemplares se depositaron en el herbario FAA (Thiers, 2019). El estudio morfológico se realizó bajo microscopio estereoscópico. Los ejemplares fueron determinados utilizando floras y otros trabajos sobre la especie (Mangieri \& Dimitri, 1961; Cozzo, 1979; Stanley \& Ross, 1986; Chippendale, 1988; Dimitri, 1988; Webb et al., 1988; Brussa, 1994). El material herborizado fue comparado con ejemplares cultivados y depositados en el herbario FAA. Asimismo, se cotejaron los ejemplares con imágenes digitales del ejemplar tipo y de otros materiales de esta especie conservados en los herbarios M y Fl. 
Se calculó el área ocupada por la población serrana en el año 2010 mediante una imagen de alta resolución espacial (Quickbird Digital Globe 10-3-2010) y clasificación orientada a objeto (OBIA) en el módulo "Feature extraction" de ENVIZOOM (ENVI 4.5); luego se comparó visualmente con la última imagen de alta resolución disponible en el sistema en línea Google Earth, correspondiente al 16/1/2016.

\section{RESULTADOS}

Eucalyptus viminalis Labill., Nov. Holl. Pl. 2: 12, tab. 151. 1806. TIPO: "Habitat in capite Van-Diemen", Australia, Labillardière s.n. (holotipo FI 011467!; isotipo M 0137424!). Fig. 2.

Árbol de gran porte, hasta alrededor de 50 $\mathrm{m}$, con la corteza lisa, caediza en largas fajas, dejando descubierto el tronco recto, blanquecino; en ejemplares poco desarrollados la corteza es rugosa y oscura. Ramas péndulas. Hojas juveniles levemente discoloras, opuestas, sésiles, generalmente amplexicaules, elípticas, lanceoladas o aovadas, de 4-10 × 1,5-3 cm. Hojas adultas concoloras, alternas, con pecíolo de 1-2 cm, acuminadas, angostamente lanceoladas hasta falcadas, de $8-20 \times 0,8-2 \mathrm{~cm}$. Flores dispuestas en inflorescencias simples, umbeliformes, axilares, generalmente trifloras (7 flores en subsp. cygnetensis Boomsma), con pedúnculo cilíndrico o aplanado, de 0,5 a $1 \mathrm{~cm}$ y pedicelos de 0,2 a $0,4 \mathrm{~cm}$. Flores con hipanto hemiesférico o campanulado, cáliz y corola soldados en el opérculo, caedizo en antesis. Opérculo hemiesférico o cónico, ligeramente apiculado, 0,3-0,4 × 0,3$0,5 \mathrm{~cm}$. Estambres numerosos, pluriseriados, largamente exsertos, blanquecinos. Filamentos filiformes, libres. Anteras ovoides, bitecas con las tecas paralelas. Ovario ínfero con los lóculos pluriovulados, placentación axilar; estilo filiforme notable. Fruto cápsula (diplotegia), hemiesférico o subgloboso, de 0,5-0,8 $\times$ 0,5-0,9 cm, dehiscente por 3-4 valvas apicales, generalmente exsertas, disco ancho, exserto. Semillas numerosas, negras, pequeñas, comprimidas, abortando muchas de ellas (las estériles son de color castaño y brillantes).
Iconografía. Labillardière (1806: tab. 151); Von Mueller (1879-1884: decade X); Dimitri (1988: fig. 194K); Brussa (1994:219).

Nombres vulgares. "Eucalipto blanco", "Manna gum", "White ribbon gum".

Distribución y hábitat. Nativa del Sudeste de Australia (Nueva Gales del Sur, Queensland, Victoria, Australia meridional) y Tasmania (Chippendale, 1988), entre $28^{\circ}$ y $43^{\circ} \mathrm{S}$, desde el nivel del mar hasta los $1500 \mathrm{~m}$ de altitud, en clima templado a templado frío (Brussa, 1994). Introducida en América del Norte, en California se ha naturalizado extensamente en algunas áreas (Ritter \& Yost, 2009). También en Hawaii (NMNH, 2019), en Nueva Zelanda, generalmente en las cercanías de los árboles plantados, especialmente después de incendios (Webb et al., 1988) y en el este de África (Verdcourt, 2001).

Si se compara con su región de origen, en cuanto a la latitud existe correspondencia entre la provincia de Buenos Aires y los territorios del sur de Australia como Victoria. Los requerimientos climáticos de esta especie se presentan en el centro de la provincia: precipitaciones (mayores a $500 \mathrm{~mm}$ anuales), temperaturas máximas promedio $\left(20^{\circ}-32^{\circ} \mathrm{C}\right)$, temperatura mínima que soporta (entre $-4^{\circ}$ y $-8^{\circ} \mathrm{C}$ y hasta $-16^{\circ} \mathrm{C}$ ) y hasta 100 heladas anuales (Mangieri \& Dimitri, 1961; Cozzo, 1979; Brussa, 1994). Por lo tanto no habría impedimentos climáticos para su establecimiento.

Los ejemplares hallados en las Sierras de Azul, Sistema de Tandilia corresponden a la subespecie viminalis, que presenta inflorescencias simples, trifloras y corteza lisa caediza; en Australia se ubica en varios ambientes húmedos, entre ellos en valles montañosos y sierras (Chippendale, 1988). Aunque ocupa suelos con relativa profundidad, se adapta a suelos pedregosos (Brussa, 1994). En Tandilia crece en roquedales (Figs. 3A, B, C); asimismo, se ha observado la presencia de árboles jóvenes a distancias cercanas a plantaciones en zonas de llanura (Fig. 3D) y en el piedemonte, donde algunos ejemplares presentan algunas inflorescencias con mayor número de flores. La población de E. viminalis ubicada sobre el Cerro San Pablo (Fig. 3E) permitiría categorizar a la especie como naturalizada en el país (Pyšek et al., 2004). El área de esta población se ha ido incrementando, alejándose de la plantación inicial, desde hace más de 30 años (Fig. 4). 

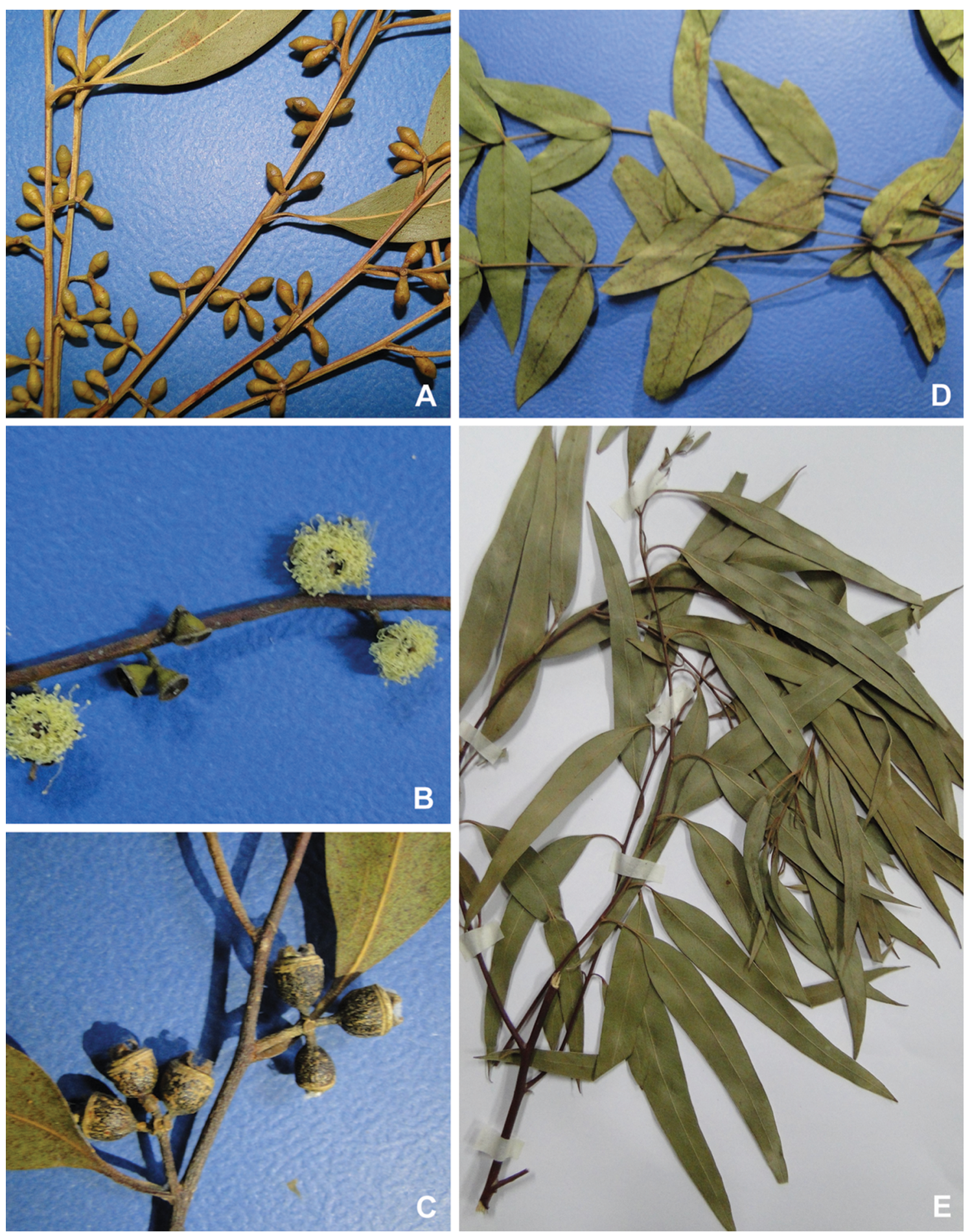

Fig. 2. Morfología de Eucalyptus viminalis. A, botones florales. B, flores. C, frutos. D, hojas juveniles. E, hojas adultas. A-C de FAA-9790, D de FAA-9974, E de FAA-3535. Fotografías: Carlos D'Alfonso. Figura en color en la versión en línea http://www.ojs.darwin.edu.ar/index.php/darwiniana/article/view/843/1175 


\section{R. L. SCARAMUZZINO ET AL. Eucalyptus viminalis Labill. en Argentina}
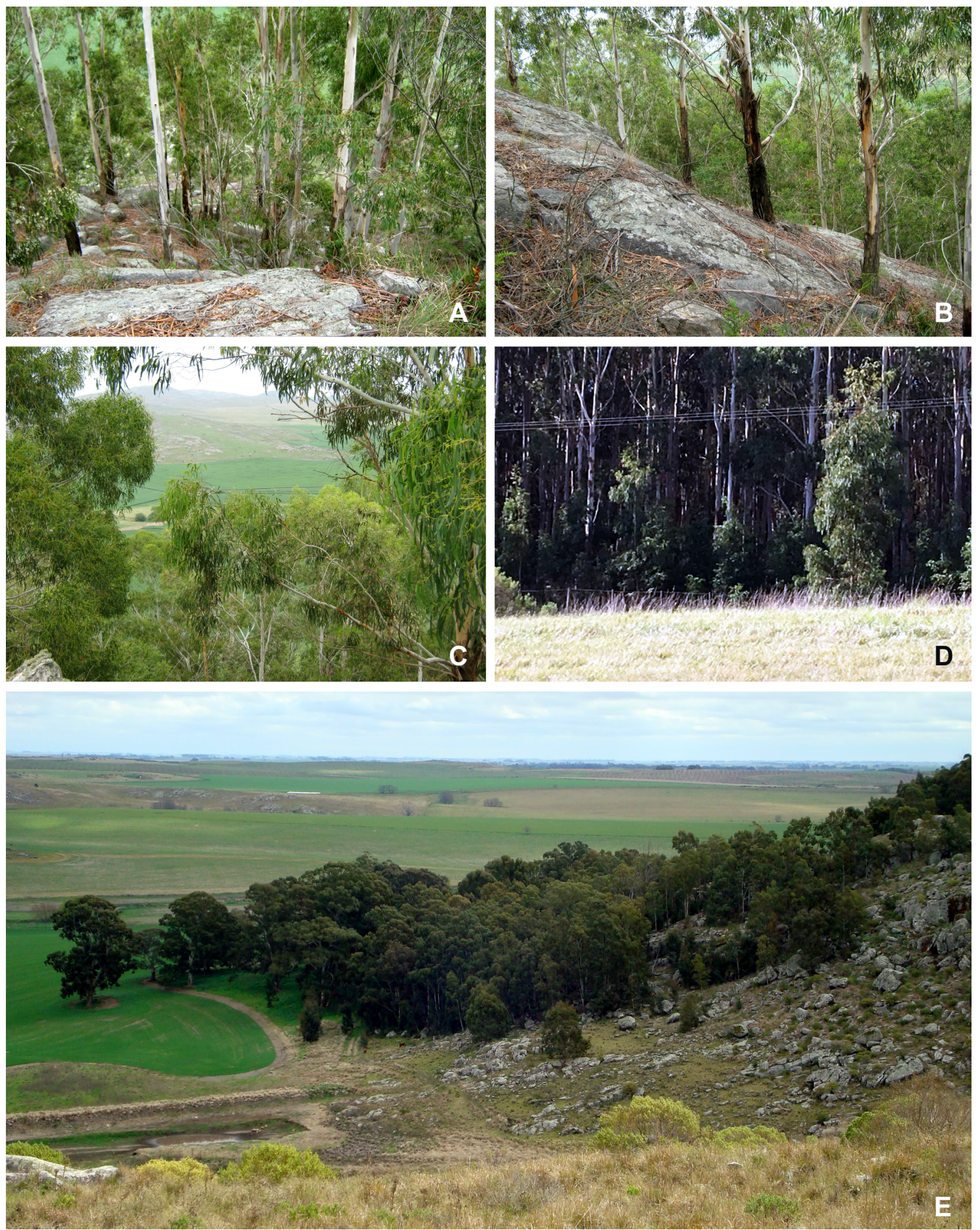

Fig. 3. Hábitat de Eucalyptus viminalis cercana al Arroyo de los Huesos en el Cerro San Pablo, Sierras de Azul, Sistema de Tandilia, provincia de Buenos Aires. A, B, C, área de roquedal (año 2009). D, área de llanura (año 2019). E, cerro San Pablo. Fotografías: Roberto Valicenti, Juan Bardi y Carlos D’Alfonso. Figura en color en la versión en línea http:// www.ojs.darwin.edu.ar/index.php/darwiniana/article/view/843/1175 


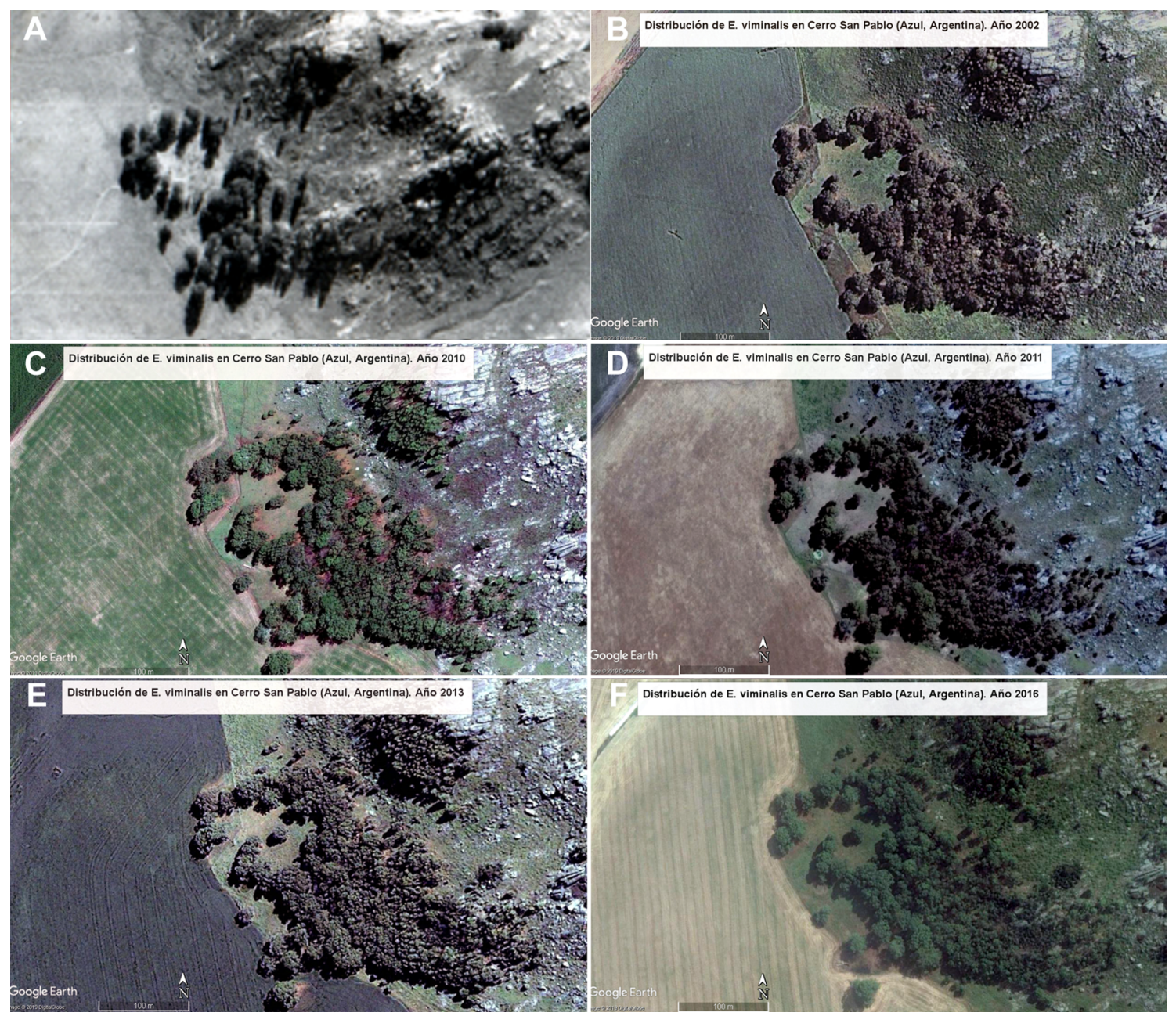

Fig. 4. Evolución de la población de Eucalyptus viminalis en Cerro San Pablo, Sierras de Azul, Sistema de Tandilia, provincia de Buenos Aires. A, fotografía aérea del año 1989. B, C, D, E y F, imágenes satelitales generadas en los años 2002, 2010, 2011, 2013 y 2016, respectivamente. Fuente: Google Earth. Figura en color en la versión en línea http:// www.ojs.darwin.edu.ar/index.php/darwiniana/article/view/843/1175

Usos. En la Argentina se ha utilizado su madera para aserrado y para trituración; para pasta para papel; para la confección de mangos de palas, escobas y otras herramientas, también cajones y otros objetos; en la construcción de viviendas, fabricación de parquet, revestimientos, terciados; para postes (se debe usar preservantes); sus flores tienen importancia en apicultura (Mangieri \& Dimitri, 1961; Cozzo, 1979). Este árbol es utilizado para cortinas de protección y sombra y puede integrar sistemas silvo-pastoriles en la producción bovina.
Observaciones. Se reproducen por semillas. Los incendios que se han producido en los pastizales serranos podrían haber contribuido a la naturalización de esta especie en sitios cercanos a las plantas madre, se favorecería la germinación $\mathrm{y}$ establecimiento de los ejemplares tanto por eliminar otro tipo de vegetación con la que competiría como por generar un ambiente propicio para la germinación de las semillas. Cozzo (1964) atribuye al fuego como el principal factor que favorece la naturalización de E. viminalis si las semillas logran germinar en época de lluvia y calor. 
Asimismo la presencia de lignotubérculos, observada tanto en ejemplares provenientes de vivero como de los repoblados, les permitiría sobrevivir a los incendios.

\section{Ampliación del área de distribución en un cerro del sistema de Tandilia}

A través de fotografías e imágenes satelitales se analizó el avance del área invadida en el Cerro San Pablo, en las Sierras de Azul, Sistema de Tandilia (Fig. 4). A partir de algunos ejemplares cultivados en la base del cerro se originó una población espontánea constituida por repoblados de diferentes edades (población disetánea). Algunos ejemplares se disponen en forma agrupada y otros aisladamente. La población se ha ido incrementando a través de los años favorecida, probablemente, por episodios de incendio. Algunos ejemplares se encuentran cerca de las plantas madres pero otros han ido ocupando el pedregal y el roquedal (Fig. 3), en competencia con la flora nativa petrofítica, donde su reconocida actividad alelopática a través de la liberación de toxinas hidrosolubles, no permite el desarrollo de ningún tipo de flora herbácea e incluso plántulas del mismo género (Granados-Sánchez et al., 2007).
En el año 2010 la población ocupaba aproximadamente 34,14 ha. En los otros sitios donde fue coleccionada, áreas de llanura y piedemonte, los ejemplares se hallan próximos a las plantas madres, ocupando áreas menores. En situaciones similares para E. camaldulensis en Santa Fe fue considerado casual (Bedetti et al., 2017). El análisis visual de la imagen de 2016 (fig. 4F), indica que no ha habido un aumento significativo en el número de ejemplares, ni en la superficie cubierta por la población. El motivo más probable es que entre las fechas de análisis no se hayan producido nuevos incendios, dado que durante la recolección a campo no se observaron indicios.

\section{Material representativo examinado}

ARGENTINA. Buenos Aires. Pdo. Azul, Sierras de Azul, Sistema de Tandilia, en cerro San Pablo, 3703'27'S 5940'26”O, 7-VIII-1999, D'Alfonso \& Farina s.n. (FAA 3535); Ruta 80, 37\%07'46,47”'S 5941'48,52'O, 28-IV-2019 (fl, fr), Bardi s.n. (FAA 9899); Ruta 60, 36 49' 48,16" S 59 28'03,34” O, 10-IV-2019 (fl, fr), Bardi s.n. (FAA 9790).

Material adicional (cultivado): Buenos Aires. Pdo. Lobería, Plantación Papelera "Mar del Plata", 26-II-1988 (fl), D'Alfonso s.n. (FAA 537). Pdo. Azul, Ea. Suerte 194, Ruta 226, 13-III-1986 (fl, fr) Borzone s.n. (FAA 5855).

\section{Clave para diferenciar las especies del género Eucalyptus naturalizadas en la República Argentina}

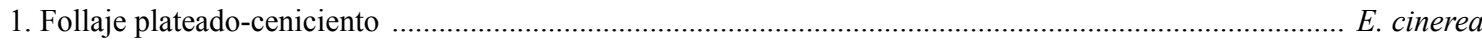

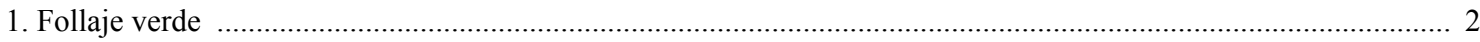

2 (1). Inflorescencias umbeliformes con flores notablemente pediceladas ……................................................... 3

2. Inflorescencias umbeliformes con flores sésiles o casi sésiles .......................................................................... 4

3 (2). Corteza persistente, rugosa profundamente surcada ................................................................. E. sideroxylon

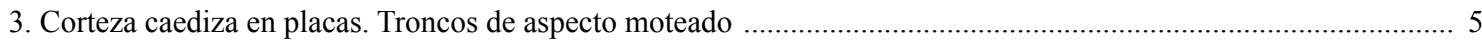

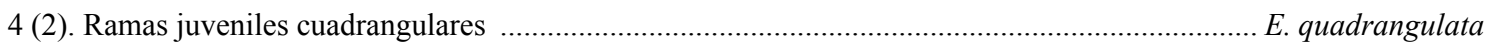

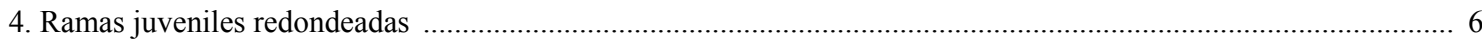

5(3). Botones florales con opérculos cónicos-corniculados ................................................................... E. tereticornis

5. Botones florales con opérculos rostrados .................................................................................. E. camaldulensis

6 (4) Botones florales glaucos. Frutos piriformes, disco incluso. Hojas juveniles pecioladas, algunos pares opuestas y luego alternas E. grandis

6. Botones florales no glaucos. Frutos hemisféricos a subglobosos, disco exserto. Hojas juveniles sésiles, opuestas 


\section{BIBLIOGRAFÍA}

Bardi, J.F; D'Alfonso, C. \& R. Scaramuzzino. 2012. Dispersión natural de dos especies de Eucalyptus en ambientes aluviales del Arroyo de los Huesos, en A. Ulberich \& M. Miranda del Fresno (eds.), Ambiente: Un compromiso de todos. Tandil: UNICEN.

Bedetti, F.; P. Rimoldi \& C. Sanabria. 2017. Eucalyptus camaldulensis Dehnh. (Myrtaceae) como una especie casual en el sur de la provincia de Santa Fe. Historia Natural, Tercera serie 7(2): 143-150.

Brussa, C. A. 1994. Eucalyptus: especies de cultivo más frecuente en Uruguay y regiones de clima templado. Montevideo: Hemisferio Sur.

Cantero, J. J.; G. E. Barboza; F. Chiarini; R. Deanna; L. Ariza Espinar; M. Giorgis; C. O. Núñez \& G. Bernardello. 2016. Novedades para la Flora de la Argentina. Boletín de la Sociedad Argentina de Botánica 51: 183-207.

Chippendale, G.M. 1988. Eucalyptus, Angophora (Myrtaceae), en A.S. George (ed.), Flora of Australia 19: 1-542. Canberra: Australian Government Publishing Service.

Cozzo, D. 1964. Ejemplos notables de crecimiento y de reproducción espontánea en Eucalyptus viminalis del partido de 25 de Mayo, provincia de Buenos Aires. Revista Forestal Argentina 8(3): 80-83.

Cozzo, D. 1976. Tecnología de la forestación en Argentina y América Latina. Buenos Aires: Hemisferio Sur.

Cozzo, D. 1979. Arboles forestales, maderas y silvicultura de la Argentina. Enciclopedia Argentina de Agricultura y Jardinería 2 (16-1): 1-256.

Dimitri, M. J. 1988. Mirtaceas, en L. R. Parodi (ed.), Enciclopedia Argentina de Agricultura y Jardinería, 3ra edición, actualizada por M. J. Dimitri, vol. 2, pp. 824-831 y 1082-1087. Buenos Aires: Ed. ACME.

FAO. 1981. El eucalipto en la repoblación forestal. Roma: Organización de las Naciones Unidas para la Agricultura y la alimentación, colección FAO Montes.

Flora Argentina, 2019. Flora Argentina: Plantas Vasculares de la República Argentina. Disponible en: http://www. floraargentina.edu.ar [Consulta abril 2019].

Granados-Sánchez, D. \& G. F. López-Ríos. 2007. Fitogeografía y ecología del género Eucalyptus. Revista Chapingo. Serie Ciencias Forestales y del Ambiente 13(2): 143-156.

Giorgis, M. \& P. Tecco. 2014. Árboles y arbustos invasores de la Provincia de Córdoba (Argentina): una contribución a la sistematización de bases de datos globales. Boletín de la Sociedad Argentina de Botánica 49: 581-603.
Henderson, L. 2007. Invasive, naturalized and casual alien plants in southern Africa: a summary based on the Southern African Plant Invaders Atlas (SAPIA). Bothalia 37(2): 215-248.

Jobbágy, E. G. \& R. B. Jackson. 2004. Groundwater use and salinization with grassland afforestation. Global Change Biology 10(8): 1299-1312. DOI: 10.1111/j.13652486.2004.00806.x

Jobbágy, E. G.; Vasallo, M.; Farley, K. A.; Piñeiro, G.; Garbulsky, M. F.; Nosetto, M. D.; Jackson, R.B \& J. M. Paruelo. 2006. Forestación en pastizales: hacia una visión integral de sus oportunidades y costos ecológicos. Agrociencia 10(2): 109-124.

Labillardière, J. J. H. de. 1806. Novae Hollandiae Plantarum Specimen volume 2. Paris: Ex Typographia Domine Huzard.

Mangieri, H. \& M. Dimitri. 1961. Los eucaliptos en la Silvicultura. Buenos Aires: ACME.

NMNH. 2019. Flora of the Hawaiian Islands. Botany, National Museum of Natural History, Smithsonian Institution, https:// naturalhistory2.si.edu/botany/hawaiianflora/ [Consulta: febrero 2019].

Paiva, J. 1997. Eucalyptus L'Hér., en S. Castroviejo, C. Aedo, C. Benedí, M. Laínz, F. Muñoz Garmendia, G. Nieto Feliner \& J. Paiva (eds.), Flora ibérica 8: 76-82. Madrid: Real Jardín Botánico - Consejo Superior de Investigaciones Científicas.

Pyšek, P.; D. M. Richardson; M. Rejmánek; G.L. Webster; M. Williamson \& J. Kirschner. 2004. Alien plants in checklists and floras: towards better communication between taxonomists and ecologists. Taxon 53: 131-143. DOI: $10.2307 / 4135498$

Rejmánek, M. \& D. Richardson. 2011. Eucalypts, en D. Simberloff \& M. Rejmánek (eds.), Encyclopedia of Biological Invasions. Berkeley and Los Angeles: University of California Press.

Ritter, M. \& J. Yost. 2009. Diversity, reproduction, and potential for invasiveness of Eucalyptus in California. Madroño 56 (3): 155-167. DOI: 10.3120/0024-9637-56.3.155

Rodriguez, R.; C. Marticorena; D. Alarcón; C. Baeza; L. Cavieres; V.L. Finot; N. Fuentes; A. Kiessling; M. Mihoc; A. Pauchard; E. Ruiz; P. Sanchez \& A. Marticorena. 2018. Catálogo de las plantas vasculares de Chile. Gayana, Botánica 75(1): 1-430. DOI: 10.4067/S071766432018000100001

Rotman, A. D. 2000. Myrtaceae, parte 1. Subfam. I. Leptospermoideae; Subfam. II. Myrtoideae, parte 1, Subtribu Myrtinae, en A.T. Hunziker (ed.), Flora Fanerogámica Argentina 70: 3-22.

SAGyP - INTA, 1995. Manual para productores de eucaliptos de la Mesopotamia Argentina. Concordia: INTA.

Stanley, T. D. \& E. M. Ross. 1986. Flora of South East Queensland, Vol. 2. Brisbane: Queensland Government. 


\section{R. L. SCARAMUZZINO ET AL. Eucalyptus viminalis Labill. en Argentina}

Steibel, P. E.; H. O. Troiani \& T. Williamson. 2000. Agregados al catálogo de las plantas naturalizadas y adventicias de la provincia de La Pampa, Argentina. Revista Facultad Agronomía Universidad Nacional de La Pampa 11(Supl 1): 75-90.

Thiers, B. [permanentemente actualizado, consulta 2019] Index Herbariorum: a global directory of public herbaria and associated staff. New York Botanical Garden's Virtual Herbarium, http://sweetgum.nybg.org/ih

Verdcourt, B. 2001. Myrtaceae, en H. J. Beentje \& S. A. L. Smith (eds.), Flora of Tropical East Africa. Rotterdam: Balkema.
Von Mueller, F. 1879-1884. Eucalyptographia: A Descriptive Atlas of the Eucalypts of Australia and the Adjoining Islands (Decades I-X). Melbourne Australia, London: J. Ferres, Government printer.

Webb, C. J.; W. R. Sykes \& P. J. Garnock-Jones. 1988. Flora of New Zealand. Vol. IV. Naturalized Pteridophytes, Gymnosperms, Dicotyledons. Christchurch: DSIR, Botany Division, Department of Scientific and Industrial Research.

Zalba, S. \& C. Villamil. 2002. Woody plant invasion in relictual grasslands. Biological Invasions 4: 55-72. 